\title{
Queuing Model for Blocking of Airport Passenger Flow
}

\author{
Xinyu Xing \\ North China Electric Power University Baoding, Baoding 071003, China; \\ 374701837@qq.com
}

\begin{abstract}
It is not a pleasant experience to queue for hours in airport security. Our mission is to model the long queuing time of airport passengers. We established queuing models to identify the bottlenecks that disrupt the passenger throughput.

We used M/M/s queuing model to analyze the per-check channel and the common channel independently. The queuing situation of zone A and zone B in each channel is also analyzed. Our model takes into account the relationship between area $\mathrm{A}$ and area $\mathrm{B}$, and expresses the average queue time of passengers in any area. Using the data given by the topic and setting the number of regional lanes, we can find the average queue time and bottleneck of traffic: under the current data conditions, the per-check channel's area A has 2 queues and area B has 5 queues, the ordinary channel is 1,5 . We compared the current checkpoint process with the bottleneck situation to point out that the current process problems.
\end{abstract}

Based on the modeling and calculation of the passenger queuing process at the airport security checkpoint, we clarified the causes of the existing problems in the airport and put forward a series of measures to solve these problems.

Keywords: Queuing Model Average Service Rate Strategy

\section{Introduction}

Airlines have a vested interest in maintaining a positive flying experience for passengers by minimizing the time they spend waiting in line at a security checkpoint and waiting for their flight. Therefore, there is a tension between desires to maximize security while minimizing inconvenience to passengers. We want to provide some creative solutions that both increase checkpoint throughput and reduce variance in wait time[1].

The current process for a US airport security checkpoint is displayed in Figure 1.

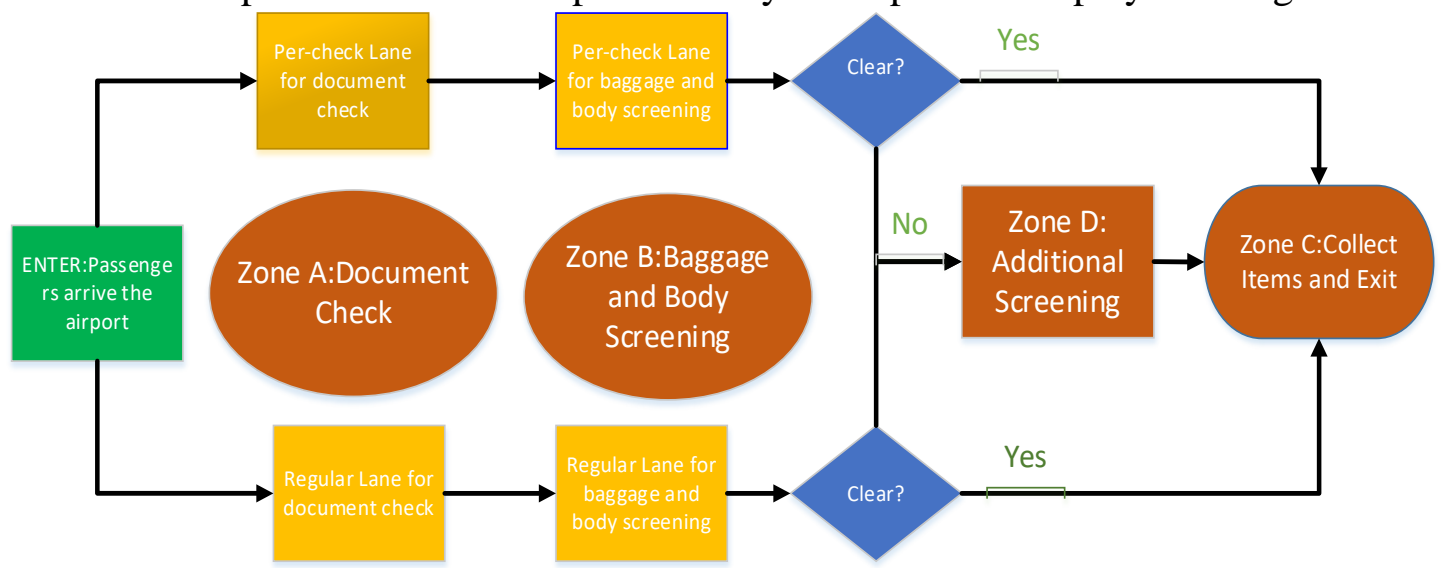

Figure 1: illustration of the TSA Security Screening Process.

We face mainly two problems:

- Explore the flow of passengers through a security check point and identify bottlenecks.

- Propose policy and procedural recommendations for the security managers based on our model. 


\section{The Queuing Model}

\subsection{Build the model}

In the discussion of the flow of passengers through a security checkpoint and bottlenecks, we consider that passengers who do not pass the microwave scanner items will enter the zone D for more inspection. Since the performance measures are components of a queuing system[2], the model can be considered as a multi-level open queuing network. Each stage can be modeled as an M/M/ S system. We divide the overall security process into primary screening and secondary screening.

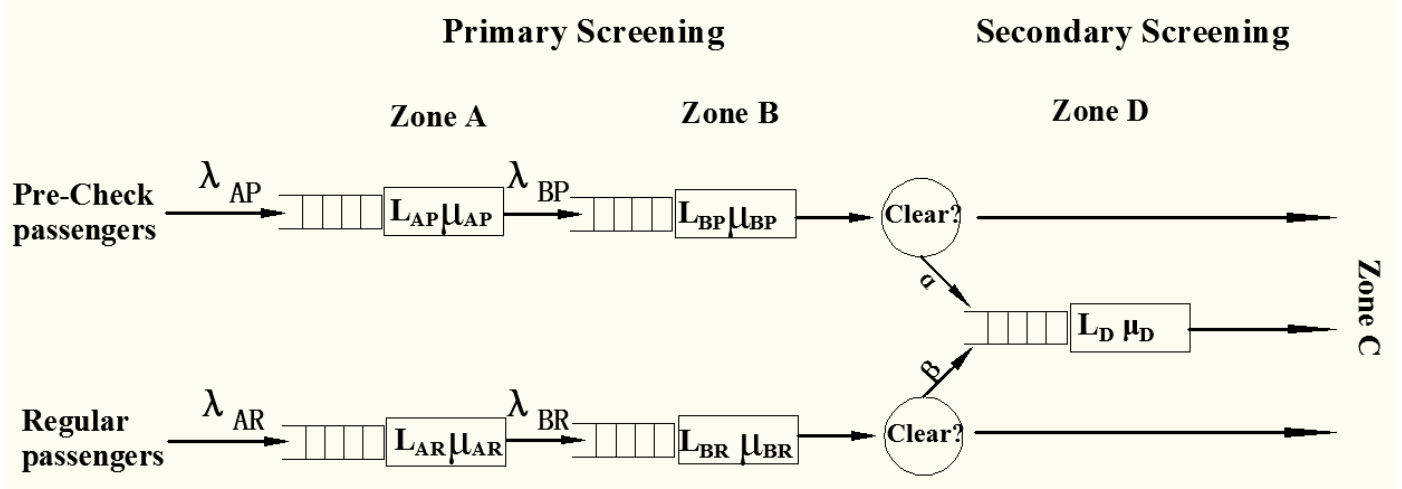

Figure 2. the overall security process

The average time a passenger spends in the system can be calculated by simply summing the average time one spends at both the primary and secondary inspection stages as depicted in E q (1):

In the primary screening:

$$
T=T_{p}+T_{S}
$$

- Passengers randomly arrive at the checkpoint, and wait in a queue, $L_{A}$, for inspecting their identification and boarding documents in zone A.

- The passengers then move to a subsequent queue for an open screening line, ${ }^{L_{B}}$.They prepare all of their belongings for X-ray screening and process through a millimeter wave in zone B.

Using the rejection rate as an indicator of accuracy. These two rate apply to determine whether the passengers to enter the queue of secondary screening, $L_{D}$.

In the secondary screening:

- Passengers that fail this step receive a pat-down inspection by a security officer in Zone D.

- Some items are flagged for additional search or screening by a security officer in Zone D.

If a passenger passes all the screenings, he or she can collect their belongings and depart the checkpoint area.

Some passengers enroll in a program called Pre-Check for trusted travelers, and enjoy a separate screening process. Thus, some parameters in the model is different from those of regular passengers, and we discuss them separately. In the last, we analysis the zone D.

\section{The Screening lane in zone $D$}

$\lambda_{D}$ is secondary arrival rate, which is directly related to the rejection rate ${ }^{\alpha}, \beta$. The rejection rate is used as an indicator of accuracy, and is related to the average time of queuing up in zone D[3]. We consider that the relationship between ${ }^{\alpha}$ and the overall security system is

$$
\alpha=\mathrm{f}\left(\max , \min , \beta^{\max }, \beta^{\min }\right)
$$

where: $\max =\max \left\{\mu_{B P}, \mu_{B R}, \mu_{D}\right\}$, $\min =\min \left\{\mu_{B P}, \mu_{B R}, \mu_{D}\right\}$,

and considered a linear curve of the form:

$$
\alpha=\left(\mu_{B P}-\min \right)\left\{\left(\beta^{\max }-\beta^{\min }\right) /(\max -\min )\right\}
$$

Assuming that $\beta^{\min }=0$ for different types of inspection methods, that is, $\mathrm{x}$-ray scanning or chemical trace, since often less intrusive processes used in primary inspections tend to have, $\beta^{\min }>0$. 
Applying the queuing model, we think, the characteristic behavior of the screening in zone $\mathrm{D}$ is a 1 to 1 single screening in a systematic manner. Thus, specific relationship and performance levels may be derived and evaluated once a particular data set is implemented in the model.

Data were not collected to indicate the specific outcome of any carry-on items that were flagged suspicious and sent to secondary inspection, that is, if any prohibited items were actually discovered or not. So we will consider the average time spending in zone D in the future work.

\subsection{Computational results and analysis}

This section reports computational results that compare the performance of the Pre-Check lanes passengers and queuing lines assignment policies with the regular lanes. For the purpose of simulating the security screening process, we consider the queuing system can finally reach the steady state, and calculate the average waiting time while the service desks different[4].

We use the resulting preprocessed data in section2 for substitution calculation. Those data are shown in the following table.

\begin{tabular}{lccc}
\hline parameter & $\begin{array}{c}\text { The arrival rate } \\
\lambda_{A}\end{array}$ & The service rate $\mu_{A}$ & The service rate $\mu_{B}$ \\
\hline Pre-Check lane & 6.65 & 5.36 & 2.13 \\
Regular lane & 4.74 & 5.36 & 1.06 \\
\hline
\end{tabular}

The computational results are shown in the following table:

Table1.Pre-check Lanes

\begin{tabular}{lllll}
\hline$s_{A P s} s_{B P}$ & $W_{A P}(\min )$ & $L_{A P}(\mathrm{n})$ & $W_{B P}(\min )$ & $L_{B P}(\mathrm{n})$ \\
\hline 1,1 & -0.9618 & -6.3957 & --- & -- \\
2,1 & 0.1167 & 0.7761 & -0.5695 & -6.1055 \\
2,2 & 0.1167 & 0.7761 & -0.5448 & -5.8405 \\
2,3 & 0.1167 & 0.7761 & -0.5703 & -6.1133 \\
2,4 & 0.1167 & 0.7761 & -0.8055 & -8.6353 \\
2,5 & 0.1167 & 0.7761 & 2.7012 & 28.9566 \\
2,6 & 0.1167 & 0.7761 & 0.2088 & 2.2378 \\
\hline
\end{tabular}

Table2.Regular Lanes

\begin{tabular}{cllll}
\hline$s_{A R}, s_{B R}$ & $W_{A R}(\min )$ & $L_{A R}(\mathrm{n})$ & $W_{B R}(\min )$ & $L_{B R}(\mathrm{n})$ \\
\hline 1,1 & 1.4263 & 6.7608 & -1.1391 & -6.1055 \\
1,2 & 1.4263 & 6.7608 & -1.0896 & -5.8425 \\
1,3 & 1.4263 & 6.7608 & -1.1405 & -6.1133 \\
1,4 & 1.4263 & 6.7608 & -1.1611 & -8.6353 \\
1,5 & 1.4263 & 6.7608 & 5.4023 & 28.9566 \\
1,6 & 1.4263 & 6.7608 & 0.4175 & 2.2378 \\
2,10 & 0.0453 & 0.2149 & 2.6037 & 27.9054 \\
\hline
\end{tabular}

In the table, negative data means that in this case service intensity is more than 1 , passenger queuing will be extremely crowded, these results are not desirable.

For the Regular lanes, in the current passenger arrival rate and airport service rate conditions, the zone A only need to open a channel. From the table 2, we find, the averaging queuing length in zone $\mathrm{A}, L_{A R}$. is 6.7608 , the averaging wait time in zone $\mathrm{A}, W_{A R \times}$ is 1.4263 . We consider that this wait time can be accepted by passengers. For zone $\mathrm{B}$, when, ${ }_{S_{A R}}=1$, the needed number of checkpoint in zone $\mathrm{B}, s_{B R}$, is 5 , and the averaging queuing length in zone $\mathrm{B}, L_{B R^{x}}$ is 28.9566 , the averaging wait time in zone $\mathrm{B}, W_{B P x}$ is 5.4023 . Obviously, this wait time can be accepted by passengers. While $s_{B R}<5$, the passengers are in a crowd state, therefore, the Regular lanes' bottlenecks appear while $s_{A R}=1$, $s_{B R}=5$. 
Finally, for the current queuing process, we can separate the fast channel and the ordinary channel $s_{A} \backslash S_{B}$ into the model solution, we obtain that the average queuing time is negative, and the two areas of the channel $B$ are in an infinite crowded state. This is in line with our conclusion that the number of channels set in zone A and B in the current queuing process has exceeded the bottleneck and the passenger queues are crowded.

\section{Conclusions}

According to our model, we propose policy and procedural recommendations as follows: When tourism is booming, the luggage of people will increase so that the average service rate will decrease. The number of channel in Zone A and B will change. According to our computation results of the model, the numbers' proportion of Zone A and Zone B in pre-check system is 1:6 and the ratio in regular is 2:6.Airport can build the standby security channel. When traffic is bigger, it would open specifically for people with disabilities. This can not only bring convenience to people with disabilities, and to avoid massive congestion .Because if some people with disabilities are checked in normal channel, it will decrease the average service rate.

\section{References}

[1] Lovell D J, Vlachou K, Rabbani T, et al. A diffusion approximation to a single airport queue[J]. Transportation Research Part C: Emerging Technologies, 2013, 33: 227-237.

[2] Martín-Cejas R R. Tourism service quality begins at the airport[J]. Tourism Management, 2006, 27(5): 874-877.

[3] Burgain P, Feron E, Clarke J P. Collaborative virtual queue: Benefit analysis of a collaborative decision making concept applied to congested airport departure operations[J]. Air Traffic Control Quarterly, 2009, 17(2): 195-222.

[4] Lee H, Simaiakis I, Balakrishnan H. A comparison of aircraft trajectory-based and aggregate queue-based control of airport taxi processes[C]//Digital Avionics Systems Conference (DASC), 2010 IEEE/AIAA 29th. IEEE, 2010: 1. B. 3-1-1. B. 3-15. 